\title{
Les quiproquos Dans Les comédies de Corneille
}

\section{Dr. Adli Abdel Raouf (*)}

\section{Les Faux Obstacles}

Les faux obstacles coupent une place très importante dans le théâtre classique. La fréquence de leur emploi est attestée par de nombreux exemples. Quand un personnage prend quelqu'un pour une autre ou quelque chose pour une autre, il se trompe. On dit qu'il y a un quiproquo ou une méprise, une équivoque, un malentendu. Le quiproquo, ressort de l'action, peut nous fournir l' 1atrigue d'une comédie vu du moins une partie de cette intrigue.

Toute la seconde moitié de LA SUIVANTE repose sur un quiproquo qui résulte d'un nom non-prononcé à la scène VII de l'acte III où GERASTE, père de DAPHNIS, croyant à tort que sa fille aime CLARIMOND, selon les dires d'AMARANTE, dit à sa fille:

\section{"Tan choix n'est pas honteux,}

Moi-même je l'agrée, et veux bien que ton âme, A ce beau cavalier ne cache plus sa flamme."

\section{(LA SUIVANTE ACT. III. SC. VII. VV 876-8)}

GERASTE n'entend que CLARIMOND, comme AMARANTE le lui fait croire à la scène précédente. Mais, comme il ne cite pas le nom de son gendre, la Jeune fille comprend que c'est de FLORAME. Tous les quiproquos qui vont suivre sont basés sur ce quiproquo entre le Père et sa fille. Ce quiproquo va Jusqu'à la scène VIII da acte v qui rassemble

(") Maître de Conférences 
tous les personnages en question: le Père, la fille et le fiancé. Lorsque GERASTE apprend à sa fille que c'est bien FLORAME qu'il lui destine Pour époux, elle s'écrire:

"Qu'un nom tu par hasard nous a donné de peine!"

(IBID. ACT. V. SC. VII v 1633

Il en résulte plusieurs équivoques: DAPHNIS, très heureuse, déclare le consentement de son père à son amant FLORAME. Celui-ci donne sa parole à DAPHNIS qui lui donne à son tour la sienne. Maintenant, il leur semble qu'il n'y a aucun obstacle à leur union :

"Nous n'avons plus qu'une âme et qu'un vouloir nous deux."

(IBID. ACT. III. SC. IX P. 432. V599.)

Mais son père n'a consenti qu'à son mariage avec CLARIMOND.

Nous avons un autre quiproquo à la scène II de l'acte IV où GERASTE apprend à sa fille qu'il lui destine un amant différent de celui qu'il lui avait naguère choisi :

"Qui dirait qu'il faut prendre un mouvement contraire, Qu'une autre occasion te donne un autre amant?»

$$
\text { (IBID. ACT. IV. SC. II VV 1046-7) }
$$

Comme GERASTE ne cite pas le nom de cet amant, DAPHNIS comprend qu'il s'agit d'un amant autre que FLORAME. Mais cette fois, il s'agit de FLORAME lui-même, alors qu'à la fois précédente il s'agissait de CLARIMOND. Là, le quiproquo est renversé et le malentendu fait l'intrigue de la comédie. Ce malentendu prolonge DAPHNIS dans 1e désespoir. Désormais, elle devient la proie d'un dilemme entre 
deux attitudes contradictoires : obéir à son Père ou respecter la parole qu'elle a donnée à son amant. Elle déclare à FLORAME

Tout amour désormais pour toi m'est interdit :

Si bien qu'il me faut être ou rebelle ou parjure, Forcer les droits d'amour ou ceux de la nature, Mettre un autre en ta place ou lui désobéir, L'irriter, ou moi-même avec toi me trahir. À moins que de changer, sa haine inévitable Me rend de tous côtés ma perte indubitable; Je ne puis conserver mon devoir et ma foi, Ni sans crime brûler pour d'autres ni pour toi.

\section{(ACT. IV. SC. VII, P443, VV 1248-56)}

Remarquons que le quiproquo a la même influence que le véritable obstacle dans le cœur de la personne qui le rencontre sur son chemin. FLORAME est tellement influencé par cette fausse nouvelle qu'il considère comme authentique qu'il va tout de suite à la recherche de son rival, d'un rival qui n'existe pas ou plutôt qui n'est autre que lui-même. Puis il pense à l'assassinat de GERASTE.

Plus j'ai d'amour pour elle, et plus pour toi de haine Enhardit ma vengeance et redouble ta peine:

Tu mourras ; et je veux, pour finir mes ennuis, Mériter par ta mort celle où tu me réduis.

(ACT. IV. SC. VIII, P445, VV 1321-24)

A la scène suivante, CELIE, confidente de GERASTE, lui apprend que son maître lui destine sa fille, mais il ne la croit pas. AMARANTE lui apprend la même nouvelle. Mais il ne la croit pas non plus. Pour dissiper tous ces malentendus, il suffit 
que FLORAME et DAPHNIS se rencontrent en présence de GERASTE. Octave Nadal a bien examiné l'atmosphère générale que Corneille a créée dans cette pièce:

"A l'avant sous un éclairage plein, un tourbillon de mensonges et de malentendus aveugle non le spectateur qui tient les ficelles et sait le fin mot de l'histoire mais les personnages eux-mêmes, les jette de surprise en surprise, les confond, les comble un instant de fausses lueurs pour les plonger de nouveaux dans les plus étranges évènements, ne sachant plus qui croire ni à qui se fier, ils en viennent à douter d'eux-mêmes de leurs propres paroles, de leurs propres jeux, ainsi poussés, ballottés de mot en mot et d'obscurités en obscurités comme en une partie de Colin- Maillard. Ils entrainent les spectateurs dans leurs vertigineuse ronde jusqu'à lui faire perdre le souffle"1

Donc, toute l'intrigue de cette comédie est fondée sur l'erreur qu'a commise GERASTE à la scène VII de l'acte III en ne citant pas le nom de son gendre. Ainsi, le quiproquo est le véritable ressort de l'action.

Dans LA VEUVE OU LE TRAITRE TRAHI, ALCIDON trompe son ami PHILISTE quand il feint d'aimer DORIS (la sœur de PHILISTE) et obtient aussi de son ami l'autorisation de se marier avec elle. Donc, PHILISTE a fait une méprise car il

1) Cf. Nadal (octave): Op. cit. P105. 
prend ALCLDON pour son ami intime et l'amant fidèle de sa sœur, mais ALCIDON n'a pour dessein que de trahir PHILISTE et de le chasser de l'esprit de CLARICE"1. Cette méprise va dès le début de la pièce jusqu'à la dernière scène de la comédie où PHILLSTE constate i infidélité d'ALCIDON. L'auteur attire lui-même l'attention sur les faux obstacles dans L'AVERTISSEMENT AU LECTEUR en tête de la comédie en question.

"Tu y reconnaitras trois sortes d'amour aussi extraordinaires au thêâtre qu'ordinaires dans le monde, celle de Philiste et Clarice, d'Alcidon et Doris, et celle de la même Doris avec Florange qui ne part Point. Le Plus beau de leurs entretiens est en équivoque, et en proposition dont ils te laissent les conséquences à tirer; si tu en pénètres bien le sens, l'artifice, ne t'en déplaira point."

PHILISTE, abusé de faux sentiments d'ALCIDON, fait une autre méprise quand il fait rompre le mariage de sa sœur avec FLORANGE que sa mère a Proposé ${ }^{3}$. Ce quiproquo va dès la scène III de 1'acte III Jusqu'à la dernière scène de la pièce.

CELIDAN a fait aussi une méprise en se laissant convaincre Par les prétextes d'ALCIDON qui le font consentir à enlever CLARICE Pour se venger de l'affront que PHILISTE

\footnotetext{
${ }^{1}$ )La Veuve ou le théâtre trahi, Acte I, sc I, et sc II.

${ }^{2}$ ) Ibid., Avertissement au lecteur, P. 203.

3 ) Ibid., Acte, III, sc. III, PP254-257.
} 
lui a fait en accordant la main de sa sœur à FLORANGE ${ }^{1}$. Mais CELIDAN, voyant de quelle manière il a été trompé, délivre CLARICE et la rend à PHILISTE. Ce quiproquo va de la scène I de 1'acte III Jusqu'à la scène $\mathrm{V}$ de l'acte $\mathrm{V}$.

Le quiproquo a pour cause quelquefois des fausses lettres. Dans LA PLACE ROYALE OU L'AMOUREUX EXTRAVAGANT, ALIDOR fait parvenir à ANGELIQUE des lettres supposées d'amour signée de son nom à CLARINE. Ces fausses lettres font passer ALIDOR pour traître aux yeux de sa maîtresse ANGELIQUE ${ }^{2}$ qui décide d'envisager le change par le change et proclame : "J'accepterai plutôt un barbare que lui " [Acte II. Sc III, P.490, v.500] Ce barbare n'est autre que DORASTE.

Dans MELITE OU LES FAUSSES LETTRES, des lettres d'amour signées du nom de MELITE à PHILANDRE font croire à l'infidélité de MELITE pour son amant TIRCIS qui prend ces lettres pour véritables. ${ }^{3}$ TIRCIS, ayant entre ses mains la preuve de la trahison de MELITE, se désespère et souhaite sa mort ${ }^{4}$. Ce quiproquo vise à contrarier un amour heureux et à faire rebondir l'action. Le naïf PHILANDRE qui se croit aimé de MELITE, va montrer ces fausses lettres à TIRCIS. Celui-ci va s'apercevoir qu'on s'est joué de Lui, et va perdre même sa véritable maitresse CLORIS (sœur de TIRCIS).

\footnotetext{
1) Ibid., Acte, III, sc. I, pp250-252.

$\left.{ }^{2}\right)$ La Place Royale ou l'amoureux extravagant, Acte II, sc. II et sc.III.

3 ) Mélite ou les Fausses lettres, Acte III, sc.II, pp47-48.

$\left.{ }^{4}\right)$ Ibid., Acte, III, sc. III, P.49- 50.
} 
Le quiproquo a pour source une fausse nouvelle. Dans MELIE OU LES FAUSSES LETTRES, LISIS, ami de TIRCIS, apprend à MELITE que celui-ci est mort à cause de son infidélité et son change. MELITE, qui prend la nouvelle pour vraie, s'évanouit.

"Hélas! Soutenez-moi, je n'en puis plus, je pâme "

\section{[Ibid., IV. Sc III, P.64]}

CLITON, voisin de MELITE, voyant la scène de l'évanouissement de MELITE conclut que celle-ci est morte. CLITON apprend à ERASTE que TIRCIS, voyant l'infidélité de son amante MELITE, est mort de douleur, et que MELITE, à son tour, après avoir appris le trépas de son amant TIRCIS, l'a suivi au tombeau'.

ERASTE, croyant que MELITE, son amante, et TIRCIS, son meilleur ami, sont morts à cause de fausses lettres, devient la proie d'un repentir si violent qu'il en devient fou ${ }^{2}$. Donc, les faux obstacles produisent les mêmes réactions dans les âmes des personnages que les vrais car ceux-ci les prennent pour vrais. Le quiproquo qui a pour source un évanouissement de certains personnages permet de montrer leurs véritables sentiments pour ceux qu'ils croient morts. Généralement, une personne évanouit est considérée comme morte. Corneille écrit dans l'Examen de sa pièce en question:

" La folie d'Eraste n'est pas de meilleure trempe. Je la condamnais dès lors en mon âme ; mais comme c'était un ornement de théâtre qui

\footnotetext{
1) Ibid., Acte, IV, sc. VI, P.66

$\left.{ }^{2}\right)$ Ibid., p66-69.
} 
ne manquait jamais de plaire, et se faisait souvent admirer, $j$ 'affectai volontiers ces grands égarements, et en tirai un effet que je tiendrais encore admirable en ce temps : c'est la manière dont Éraste fait connaître à Philandre, en le prenant pour Minos, la fourbe qu'il lui a faite, et l'erreur où il l'a jeté"'1.

La crise de la folie d'ERASTE va de la scène VI de l'acte IV Jusqu' à la scène II de l'acte V.

Nous trouvons, chez Corneille, des quiproquos ayant pour cause une erreur sur la Personne. Dans MELIE OU LES FAUSSES LETTRES ERASTE, dans sa folie prend la nourrice pour sa maîtresse MELITE ${ }^{2}$. Mais ce quiproquo ne dure pas longtemps. Nous trouvons ce quiproquo dans LA PLACE ROYALE OU L'AMOUREUX EXTRAVAGANT. ALIDOR persuade ANGELIQUE de s'enfuir avec lui pour éviter le mariage qu'elle a promis, malgré elle, à DORASTE. Mais, au fond de son âme, ALIDOR ne vise qu'à faire enlever ANGELIQUE Par son ami CLEANDRE qui profitera Luimême de cet enlèvement et aura ANGELIQUE. ALIDOR, "sortant de la porte d'ANGELIQUE, et repassant sur le théâtre," : proclame:

"Cléandre, elle est à toi, J'ai fléchi son courage.

Que ne peut l'artifice, et le fard du langage!

Et si pour un ami ces effets Je produis,

\footnotetext{
$\left.{ }^{1}\right)$ Mélite ou les Fausses lettres, Examen, P 6-7.

) Ibid., Acte, V, sc. II, P.76
} 
Lorsque j'agis pour moi, qu'est-ce Je ne puis?

\section{(LA PLACE ROYALE ACT. III. SC. VI P. 505. VV 905-8)}

A la troisième scène de l'acte IV, ALIDOR "lui donne la promesse de Cléandre" et ANGELIQUE, la Prend sans lire et lui dit:

Je prends sans lire, et ta roi est si claire

Que je la prends bien moins pourquoi que Ron reprend Père

Je la porte la chambre, épargnons les discours.

Fais avancer les gens, et dépêche.

\section{(IBID. ACT. IV. sc. III P, 510-11 v 1029 32)}

ANGELIQUE sort de la scène pour aller à sa Porte l'aident mais PHYLIS, sœur de DORASTE, la suit et la Surveille secrètement Alidor paraît avec Cléandre bien accompagne d'une troupe et après lui avoir montré tombe Phyllis qu'il croit être ANGELIQUE, il se retire en Son un coin du théâtre, et Cléandre enlevé Phyllis, et lui met d'abord la main sur la bouche ${ }^{1}$. Le ALIDOR est étonné de voir ANGELIQUE qu'il croyait être enlevé par CLEANDRE ${ }^{2}$. Cet enlèvement par erreur sur l'identité de la personne sera suivi par le mariage de CLEANDRE avec Phyllis. Il est vraiment pour lui donner moyen avoir la main d'ANGELIQUE, cependant, il n'atteint pas ce but. Pour ALIDOR, cet enlèvement est un moyen de se débarrasser d'ANGELIQUE et de reprendre sa liberté.

1) La Place Royale ou l'amoureux extravagant, Acte IV, sc. IV P. 511.

) Ibid., Acte, IV, sc. VI, P.513 
Le quiproquo qui a pour cause l'ignorance de identité de la personne aimée se trouve dans LA SULLE Du MENTEUR ou DORANTE ignore l'identité de la belle inconnue qui s'intéresse lui et dont il tombe amoureux, sans savoir son nom ni connaitre son visage. Il est curieux de connaitre cette inconnue, mais sa curiosité est mêlée de et perplexité: il dit à son valet CLITON en lui montrant le portrait de cette inconnue.

"Viens, Cliton et regarde. Est-elle vieille ou laide?

Voit- on des yeux plus vifs? Voit-on des traits plus doux?"

\section{(LA SUITE DU MONTEUR II, SC. VII VV 760-61)}

DORANTE ne voudrait pas trahir son ami CLEANDRE dont l'inconnue est peut-être la maîtresse ou sa femme parce qu'elle habite chez lui ${ }^{1}$. Puis DORANTE déclare son amour à la belle inconnue sans l'avoir reconnue, car celle-ci, voilée, se fait passer pour une sœur de LYSE, femme de chambre de MYLISSE. DORANTE dit à LYSE qui lui demande de rendre le portrait de MYLISSE:

" Va dire de ma mort à celle qui t'envoie ;

Qu'il fait tout mon bonheur, qu'il fait toute ma joie,

Que rien n'approchait de mon ravissement,

Si je le possédais de son consentement ;

Qu'il est l'unique lin où mon espoir se fonde;

Qu'il est le seul trésor qui me soit cher au monde."

(IBID, ACT. III, SC.III, P145-6 VV 989-94)

$\left.{ }^{1}\right)$ La suite du Menteur, Acte, IIi, sc. II, Pp.141-142 
MELISSE, déguisée en servante, lui déclare aussi son amour.

" Si vous l'aimez, Monsieur, croyez qu'en son courage,

Elle vous aime assez, pour vous laisser ce gage."

(IBID. P. 147, VV 1021-22)

Puis ravie d'une telle épreuve d'amour, MELISSE se découvre et DORANTE reconnaît que c'est elle-même qui lui a envoyé de l'argent, la lettre et le portrait et " loge en Bellecour."1

Mais DORANTE ne découvre que cette belle est la sœur de CLEANDRE qu'à la fin de la pièce. Dans cette comédie, si DORANTE tome amoureux de MELISSE sans l'avoir vue, celle-ci, après la description que LYSE lui a faite DE DORANTE, ressent pour lui, un amour très vif avant même de l'avoir vu'.

Si nous trouvons ci-dessus dans la suite du Menteur un quiproquo dû à l'ignorance de l'identité de l'amante inconnue, nous trouvons dans Don Sanche d'Aragon le quiproquo dû à l'ignorance de l'identité de l'amante inconnu. Dans cette pièce, la reine D. Isabelle aime Carlos, cavalier inconnu dont on ignore l'identité et l'origine; son amour pour Carlos lui crée des situations embarrassantes. Comment une reine peut-elle se marier avec un cavalier inconnu? Elle devient la proie d'un combat intérieur entre deux attitudes inconciliables: entre son

\footnotetext{
$\left.{ }^{1}\right)$ Ibid., Acte, III, sc. III, PP.148-149

$\left.{ }^{2}\right)$ Ibid., Acte, II, sc. I, PP.120-123
} 
amour pour Carlos et sa dignité comme reine. ${ }^{1}$ Elle tente de le rapprocher d'elle en le mariant avec la sœur de celui qu'elle va épouser elle-même. Elle dit à D. Manrique et D. Lope dont elle va choisir l'un comme mari:

" En un mot, vous avez l'un et l'autre une sœur

Et je veux que le Roi qu'il me plaira de faire

En recevant ma main, le fasse son beau-frère."

\section{(ACT. III, SC.IV, P590, VV 908-10.)}

Mais elle apprend à la fin, que ce Carlos n'est autre que D. Sanche d'Aragon, et par conséquent il est digne de sa main.

De même, dans cette pièce, D. Elvire, princesse d'Aragon aime Carlos, et le préfère à D. Elvire. Comment ose-t-elle se marier avec un simple cavalier fils d'un pêcheur dont on ignore l'origine et l'identité? Mais à la fin, elle découvre que ce Carlos n'est autre que son frère, D. Sanche d'Aragon. Carlos, aussi, depuis son apparition à la scène III de l'acte I, se croit fils d'un pêcheur; tous les autres personnages de la pièce le croient comme tel. Il ne sait pas que D. Elvire qui partage son cœur avec D. Isabelle est en réalité sa sœur. Ce malentendu va jusqu'à la dernière de la pièce où Carlos reconnait qu'il est Don Sanche d'Aragon et frère d'Elvire, et il est digne de la main de D. Isabelle. Enfin, D. Isabelle, D. Elvire, D. Léonor, se trompent sur l'identité de Carlos, mais celui-ci se trompe non seulement sur l'identité des autres personnages, mais aussi sur sa propre identité. Disons que l'intrigue de cette pièce est fondée sur ce malentendu.

$\left.{ }^{1}\right)$ Don Sanche d'Aragon, Acte, III, sc. IV, PP.33-38 
LE MENTEUR est fondé tout entier sur l'erreur qu'a commise DORANTE a la scène IV de l'acte I sur l'identité des deux jeunes filles rencontrées aux Tuileries: CLARICE et LUCRECE. Il croit que la plus belle de ces jeunes filles qui lui a parlé et qui a su le charmer, s'appelle LUCRECE, mais vraiment la plus belle et dont il tombe amoureux, s'appelle CLARICE. Donc, il prend LUCRECE pour CLARICE. Désormais, il n'échappe à un malentendu que pour tomber dans un autre et les mensonges auxquels il est entraîné naissent de cette erreur. Ce quiproquo va jusqu'à la scène VI de l'acte $V$ où DORANTE s'aperçoit de son erreur.

Un double quiproquo s'établit entre DORANT et son père GERONTE. DORANTE, croyant que la femme qu'il aime et qu'il désire s'appelle LUCRECE, fait croire à son père qu'il est déjà marié à Poitiers. Il pense ainsi d'éviter le mariage avec CLARICE que son père veut lui imposer. Alors, son père ne cherche à le marier qu'avec celle qu'il aime. GERONTE, prenant le mariage de son fils à Poitiers pour vrai, comme ce dernier lui fait comprendre, est abusé par ce mensonge et va se dégager du père de CLARICE ${ }^{1}$. Constatons que sans le malentendu que DORANTE a commis au premier acte (prendre LUCRECE pour CLARICE), notre pièce se terminerait à la fin du troisième acte par le mariage de DORANTE avec CLARICE car le père de DORANTE et celui de CLARICE ont presque déjà conclu l'affaire entre eux.

$\left.{ }^{1}\right)$ Le Menteur, Acte, II, sc. V, PP. 33-38. 
Donc, le quiproquo peut résulter d'une confusion de nom. Un personnage, croyant parler d'une personne, en désigne effectivement une autre. DORANTE croit que CLARICE, la jeune fille dont il est épris, à laquelle il a parlé et qui l'a su charmer, se nomme LUCRECE. De plus, plusieurs faits l'enfoncent dans son erreur, CLARICE lui donne un rendezvous sous la fenêtre de son amie LUCRECE et le billet qu'elle envoie pour limiter le rendez-vous est signé du nom de LUCRECE $^{1}$. CLARICE, se faisant passer pour LUCRECE, parle à DORANTE dans l'obscurité. Celui-ci, persuadé à présent plus que jamais que la femme dont il est épris s'appelle LUCRECE, déclare à CLARICE son amour et son désir de se marier avec elle. Mais c'est impossible puisqu'il est déjà marié à Poitiers. DORANTE nie ce mariage et déclare qu'il est prêt à l'épouser dès le lendemain. Il ne prétend qu'il est marié que pour éviter un mariage avec une autre. Puis il déclare qu'il n'aime que LUCRECE et n'a que du mépris et des dédains pour CLARICE. Ce qui le brouille avec celle-ci ${ }^{2}$ qui va rentrer à ALCIPPE. Puis DORANTE fait une cour assidue à LUCRECE qu'il n'aime pas.

Pour se débarrasser de son père qui voudrait voir sa bellefille, DORANTE fait croire à son père que sa femme est enceinte et qu'elle ne peut pas venir le voir. Le père, très heureux, veut écrire au père de sa belle-fille, mais DORANTE oublie le nom qu'il avait déjà dit à son père et lui dit un autre

\footnotetext{
1) Ibid., Acte, II, sc. VII, P. 39.

2) Ibid., Acte, III, sc. V, PP. 48-54.
} 
nom. PYRANDRE, le père se souvient d'avoir jadis entendu un autre nom, c'est ARMEDEON. Son fils persuade que son père se sert de ces deux noms ${ }^{1}$. Ce malentendu ne dure qu'à la première scène de l'acte $\mathrm{V}$ où GERONTE apprend de PHILISTE- auprès duquel il va se renseigner à propos de la femme de son fils- que son fils est menteur. Le père va prendre son fils au piège. Celui-ci qui n'a d'autres inventions à faire, avoue la vérité à son père. C'est par amour pour LUCRECE qu'il est obligé de mentir afin d'éviter le mariage avec CLARICE qu'il n'aime pas. Il implore son père de l'aider dans son mariage avec celle qu'il aime.

Le choix que vos bontés avaient fait de Clarice,

Sitôt que je le sus, me parut un supplice;

Mais comme j'ignorais si Lucrèce et son sort

Pouvaient avec le vôtre avoir quelque rapport,

Je n'osai pas encore vous découvrir la flamme

Je vous conjurerais, par les nouds les plus doux

Dont l'amour et le sang puissent m'unir à vous,

De seconder mes voux auprès de cette belle:

Obtenez-la d'un père, et je l'obtiendrai d'elle.

\section{(ACT V, SC.III, P 78, VV. 1565-9, VV. 1774-}

8.)

Le père, toujours tolérant, va faire son possible pour arranger le mariage de son fils avec LUCRECE :

"Je veux encore un coup montrer un cour de père,

Je veux encore un coup pour toi me hasarder,

\footnotetext{
1) Ibid., Acte, IV, sc. IV, PP. 61-63.
} 
Je connais ta Lucrèce, et la vais demander,

Mais si de ton côté le moindre obstacle arrive..."

\section{(IBID, P.79 VV 1586-89)}

Dans cette comédie, nous trouvons aussi un quiproquo entre ALCIPPE et sa maîtresse CLARICE. C'est sous l'effet de mensonge de DORANTE à propos de la fête sur l'eau qu'ALCIPPE accuse sa maîtresse d'avoir passé la nuit avec DORANTE. Mais CLARICE lui assure qu'on lui joue un mauvais tour.

Quelqu'un a pris plaisir à se jouer de vous Alcippe, croyez-moi"

\section{(ACT II, SC.III, P 31, VV. 520-21)}

Mais ce malentendu ne va qu'à la première scène de l'acte III où ALCIPPE apprend de PHILISTE que DORANTE n'est qu'un menteur et la fête sur l'eau imaginaire. Désormais, ALCIPPE, éclairé ne se laisse pas prendre aux inventions de DORANTE.

Dans cette comédie, personne n'a échappé à la méprise, même les personnages secondaires. CLITON, surprenant la conversation où DORANTE ment sur son père et lui raconte l'histoire de son mariage à Poitiers est dupé lui-même par ce mensonge et le prend pour vrai ${ }^{1}$. Mais ce malentendu ne dure qu'une seule scène et DORANTE dévoile lui-même le secret à son valet :

"Ô l'utile secret que mentir à propos !"

(IBID, IV SC. II, V 1164)

1) Ibid., Acte, II, sc. V, PP. 35-38. 
CLITON a fait une autre méprise à la scène I de l'acte IV en croyant qu'ALCIPPE a été vraiment tué par DORANTE comme l'affirme ce dernier. Mais ce quiproquo ne dure pas longtemps. CLITON, ayant la surprise de voir ALCIPPE devant lui, dit à son maître :

"Les gens que vous tuez se portent assez bien."

\section{(IBID, IV SC. II, V 1164)}

Malgré tout, Cliton se laisse tromper encore par l'artifice de Dorante qui lui parle:

"D'une source de vie"

Que nomment nos guerriers poudre de sympathie"

\section{(IBID, Acte IV SC. III, p.60 VV. 1181-82)}

Cliton, croyant qu'il y a vraiment une poudre extraordinaire qui ressuscite les morts, dit à son maitre:

"Donnez- m'en le secret, et je vous sers sans gage"

\section{(IBID, V 1194)}

CORNEILLE a fait une place à la méprise sur les mots et il tire des effets comiques. Elle peut se présenter, chez lui, sous deux formes. Tantôt l'équivoque est volontaire ; celui qui parle le fait exprès d'une façon ambiguë. Dans ce cas, le spectateur apprécie l'adresse de celui qui fait le quiproquo et se moque de celui qui est dupé. Tantôt, l'équivoque est involontaire, il arrive qu'un personnage se méprenne sur la portée de ses paroles et dise par exemple la vérité sans s'en rendre compte.

Parmi l'équivoques volontaire, signalons les expressions à double sens qu'un personnage utilise pour se moquer de son 
interlocuteur qui ne le comprend pas. À la scène V de l'acte V de LA SUITE DU MENTEUR, PHILISTE dit à DORANTE :

"Rentrez dans la prison dont vous vouliez sortir."

(LA SUITE DU MENTEUR, ACT. V, SC. 5

\section{1844)}

PHILISTE répète ce vers trois fois. DORANTE, croyant que PHILISTE veut se venger de lui et ne veut pas sa libération, s'en désespère. PHILISTE lui explique que la prison cette fois n'est que " Le nœud de l'hyménée" [v. 1888] avec sa maîtresse MELISSE. Nous trouvons un quiproquo du même genre à la scène VIII de l'acte IV de L'ILLUSION COMIQUE où le geôlier apprend à CLINDOR qui est en prison qu'il mourra dans la nuit; le geôlier entend qu'il s'échappera de prison la nuit ${ }^{1}$. Mais CLINDOR comprend qu'il sera exécuté ; puis à la scène suivante, le geôlier le fait sortir de la prison. ISABELLE qui l'attend à l'extérieur s'enfuit avec lui avec grande joie ${ }^{2}$. A la scène $\mathrm{V}$ de l'acte III de cette même pièce, Clindor dit à Lyse servant d'Isabelle;

"Vous partagez vous deux mes inclinations:

J'adore sa fortune et tes perfections"

\section{(IBID, P 647 VV. 783-4)}

Lyse comprend que Clindor l'aime en réalité, mais celui-là n'aime qu'Isabelle. Il dit à Lyse

" Je suis dans la misère, et tu n'as point de bien;

Un rien s'assemble mal avec un autre rien"

(IBID, VV. 7891-92)

\footnotetext{
$\left.{ }^{1}\right)$ l'Illusion comique, Acte, IV, sc. VIII, PP. 669-670.

2) Ibid., Acte, IV, sc. IX, PP. 670-671.
} 
LYSANDRE de LA GALERIE DU PALAIS dit en rencontrant CELIDEE et HIPPOLYTE à la scène V de l'acte III

"Merveille des beautés, seul objet qui s'engage..."

\section{(LA GALERIE DU PALAIS, ACT. III, SC.V, \\ P. 341 V 857)}

CELIDEE, croyant que ces paroles s'adressent à elle, dit :

"N'oublierez-vous jamais cet importun langage?

Vous obstinez encore à me persécuter."

\section{(IBID, VV 858-9)}

Mais LYSANDRE, pour exciter la jalousie de CELIDEE, affirme qu'il parle à HIPPOLYTE :

" À présent que mon cour adore une autre Reine

A présent qu'Hippolyte en est la souveraine..."

\section{(IBID, P. 342, VV 889-90)}

Un autre genre de quiproquo s'établit entre la comédie et nous les spectateurs. A la fin de l'illusion comique, nous croyons, nous spectateurs, ainsi que Pridamant père de Clindor qui n'est qu'un des spectateurs, parmi nous, que Clindor est mort, comme nous l'avons vu, nous avons la grande surprise, et les autres comédiens se partager de l'argent. Il ne jouait qu'un rôle de théâtre. Cette erreur n'occupe que deux scènes.

Enfin, constatons que le théâtre comique de Corneille est plein de quiproquo. L'auteur aime mieux ce moyen dramatique, comme ressort de l'action. L'intrigue de certaines de ses comédies est fondée toute entière sur des quiproquos par exemple celle de la $\underline{\text { Suivante, }}$ celle du Menteur. Si le quiproquo 
ne lui fournit pas l'intrigue de la comédie entière, il lui en donne au moins une partie.

\section{Bibliographie.}

- Corneille, œuvres complètes, I,I textes établis, présentés et annotés par Georges Couton," Bibliothèques de la Pléiade" Paris, Gallimard, 1980.

Mélite ou les Fausses Lettres, 2-89 P.

La Veuve ou le théâtre trahi, $200-289$ P.

La Galerie du Palais ou l'Amie Rivale, 300-381 P.

La suivante, 382-459 P.

La Place Royale ou l'Amoureux Extravagant, 468$531 P$.

L'Illusion Comique, 611-688 P.

- Corneille, œuvres complètes, I,I textes établis, présentés et annotés par Georges Couton," Bibliothèques de la Pléiade" Paris, Gallimard, 1984.

Le Menteur, 2-91 P.

La Suite du Menteur, $92-181 P$.

Don Sanche d'Aragon, 547-621 P.

\section{Etudes sur l'homme et sur l'œuvre}

- Bertaud (Madeleine),

"La Place Royale ou le jaloux extravagant" dans Pierre Corneille, Actes du colloque tenu à Rouen du 2 au 6 octobre 1984, PP325-343.

- Blan (André) 
" A propos de "l'illusion comique ou sur quelques hauts secrets de pierre Corneille" dans Revue d'histoire du théâtre, 1984-II, PP207-217

- Brasillach (Robert)

Corneille, l'homme et son ouvre, Paris, Artheme Fayad, 1938.

- Dort (Bernaud)

Corneille dramaturge, Essai (travaux18), Paris, L'Arche, 1972, $171 P$.

- Herlant (Louis),

Corneille par lui-même, "Ecrivains de toujours" Paris, ed. du seuil, 1956, 192 P.

- Huszar (Guillaume), Pierre Corneille et le théâtre espagnol, Paris, Bouillon, 1903.

- Cosnier (Colette),

" Un étrange monstre, l'illusion comique", Europe, avril-mai, 1974 PP. 103-133

- Cosnier (Colette),

Corneille, L'Illusion comique, Paris, Bordas, 1985, $128 \mathrm{p}$.

- Lemonnier (Léon)

Corneille, Paris, Jules Taillandier, 1945 
